\title{
RANDOM MARKED SETS
}

\author{
F. BALLANI, ${ }^{*}$ TU Bergakademie Freiberg \\ Z. KABLUCHKO, ${ }^{* *}$ Universität Ulm \\ M. SCHLATHER, ${ }^{* * *}$ Universität Göttingen
}

\begin{abstract}
We aim to link random fields and marked point processes, and, therefore, introduce a new class of stochastic processes which are defined on a random set in $\mathbb{R}^{d}$. Unlike for random fields, the mark covariance function of a random marked set is in general not positive definite. This implies that in many situations the use of simple geostatistical methods appears to be questionable. Surprisingly, for a special class of processes based on Gaussian random fields, we do have positive definiteness for the corresponding mark covariance function and mark correlation function.
\end{abstract}

Keywords: Random field; random set; marked point process; mark correlation function; mark covariance function

2010 Mathematics Subject Classification: Primary 60G60; 60G55

Secondary 60G15; 60D05

\section{Introduction}

Quantities measured in space are mostly modelled as so-called regionalized variables under the implicit assumption that these quantities can, in principle, be measured everywhere and that the choice of sampling points does not depend on the values of these quantities. Based on this assumption, several geostatistical methods like variogram analysis or kriging can be applied [6]. However, there are two types of situation where this assumption does not hold [24] and, hence, uncritical use of geostatistical methods might result in incorrect or meaningless results.

The first type of problem is caused by the investigators themselves following some kind of preferential sampling [9]. For instance, this happens when data are sampled at places where only high values of the variable of interest are expected. The second type of problem is intrinsic to the investigated object itself. An obvious situation is the investigation of individuals, e.g. trees in a forest, where interactions among individuals are present. In this particular situation the theory of marked point processes provides a formal framework for data analysis [10], [13].

In this paper we draw the reader's attention to some further, deceptive situations which belong to the second type of problem and where implicit conditioning has mostly been ignored in the literature [14, p. 157], [16], [29]. For instance, the investigation of pesticides in soil is restricted to cropland and the height of forest litter is restricted to silvicultural areas. In both cases, an unintended preselection cannot be excluded since environmental conditions directly

\footnotetext{
Received 2 December 2009; revision received 2 February 2012.

* Postal address: Institut für Stochastik, TU Bergakademie Freiberg, D-09596 Freiberg, Germany.

Email address: ballani@math.tu-freiberg.de

** Postal address: Institut für Stochastik, Universität Ulm, Helmholtzstr. 18, D-89069 Ulm, Germany.

Email address: zakhar.kabluchko@uni-ulm.de

*** Current address: Institut für Mathematik, Universität Mannheim, A5, 6, D-68131 Mannheim, Germany.

Email address: schlather@math.uni-mannheim.de
} 
influence land use. A further, simple example which has motivated this work is the prediction of the altitude by geostatistical methods based on measurements that are taken above sea level only.

Such conditioning might be considered minor, but can cause major effects, nonetheless. We advise caution because of the following facts.

- Any characteristic, such as the covariance function or the variogram, has to be understood as a conditional quantity, given that measurements can be taken at certain locations.

- In general, the covariance function is not positive definite and the variogram is not conditionally negative definite.

Since Gaussian random fields are rather popular, a bigger part of this paper deals with the following hypothetical model: the sea level is at 0 and the altitude is given by some (smooth) stationary Gaussian random field $Z$ with mean $-t$ and variance 1 . Then we face the following oddities when inference is based on measurements above sea level only.

1. The theoretical variogram is not conditionally negative definite, in general.

2. The naive definition of the covariance function

$$
C(x, y)=\mathrm{E}[Z(x) Z(y) \mid Z(x) \geq 0, Z(y) \geq 0]-\bar{m}^{2}
$$

leads in general to a function that is not positive definite for any $\bar{m} \in \mathbb{R}$.

3. A more suitable definition of the covariance function for the altitude above sea level as the conditional covariance given that $Z(x) \geq 0$ and $Z(y) \geq 0$ leads to a function that is never differentiable unless the field is spatially constant.

4. If $t=0$, the conditional covariance function is positive definite, although no random field exists that is independent of the sampling locations and that can model the altitude above sea level.

Before discussing the above setup in detail, we introduce the theoretical framework that allows both a meaningful definition of second-order characteristics and usual random fields as well as the inclusion of marked point processes as particular cases. For this reason, we extend the notion of a random upper semicontinuous (u.s.c.) function (taking values in $\overline{\mathbb{R}}=[-\infty, \infty]$ ) on $\mathbb{R}^{d}$ such that the domain is a random subset of $\mathbb{R}^{d}$. To this end, we make use of Matheron's [17] idea and consider the hypograph

$$
A_{f}=\{(x, t) \in X \times \overline{\mathbb{R}}: t \leq f(x)\}, \quad X \subseteq \mathbb{R}^{d},
$$

of a function $f: X \rightarrow \overline{\mathbb{R}}$. In fact, $A_{f}$ is closed if and only if $f$ is u.s.c. on closed $X$, and the mapping $f \mapsto A_{f}$ is a bijection.

The paper is organized as follows. In Section 2 we formally introduce the notion of a random marked closed set and discuss some examples. In Section 3 we generalise the definitions of several characteristics of random fields and random marked sets. We show that, in general, they do not share the same definiteness properties as their random field analogues. In Section 4 we study Gaussian random fields $Z(x)$ given that $Z(x)$ exceeds a certain threshold $t \in \mathbb{R}$. In Section 5 we collect the proofs of the statements of the preceding sections. 


\section{Random marked closed sets}

Denote by $\overline{\mathbb{R}}=\mathbb{R} \cup\{-\infty,+\infty\}$ the extended real line. Let

$$
\Phi_{\text {usc }}=\left\{(X, f): X \subseteq \mathbb{R}^{d} \text { is closed, } f: X \rightarrow \overline{\mathbb{R}} \text { is u.s.c. }\right\} .
$$

Observe that $\Phi_{\text {usc }}$ is isomorphic to the system $u_{\mathrm{cl}}$ of all closed sets $A \subseteq \mathbb{R}^{d} \times \overline{\mathbb{R}}$ which satisfy, for all $x \in \mathbb{R}^{d}$ and all $t \in \overline{\mathbb{R}}$,

$$
(x, t) \in A \quad \Longrightarrow \quad\{x\} \times[-\infty, t] \subseteq A
$$

by the bijection

$$
\begin{aligned}
\tau: \Phi_{\mathrm{usc}} & \rightarrow \mathcal{U}_{\mathrm{cl}} \\
(X, f) & \mapsto\{(x, t) \in X \times \overline{\mathbb{R}}: t \leq f(x)\}, \quad(X, f) \in \Phi_{\mathrm{usc}} .
\end{aligned}
$$

The subsequent proposition follows immediately from the fact that the space $\mathcal{F}\left(\mathbb{R}^{d} \times \overline{\mathbb{R}}\right)$ of closed subsets of $\mathbb{R}^{d} \times \overline{\mathbb{R}}$ is compact [17], [20, p. 399] and that $\mathcal{U}_{\mathrm{cl}}$ is closed in $\mathcal{F}\left(\mathbb{R}^{d} \times \overline{\mathbb{R}}\right)$.

Proposition 1. $\Phi_{\mathrm{usc}}$ is compact in the topology induced by $\mathcal{u}_{\mathrm{cl}}$.

Definition 1. Let $(\Omega, \mathcal{A}, \mathrm{P})$ be a complete probability space, and let $(\Xi, Z): \Omega \rightarrow \Phi_{\text {usc }}$ be a mapping with

$$
\{\omega \in \Omega: \tau((\Xi, Z)(\omega)) \cap B \neq \varnothing\} \in \mathcal{A}
$$

for every compact set $B$ in $\mathbb{R}^{d} \times \overline{\mathbb{R}}$. Then $(\Xi, Z)$ is called a random marked closed set.

The distribution law of a random closed set is characterized by the probabilities of hitting compact sets [18], [20] whereas, by [18, Proposition 2.3.1], it suffices to consider a suitable base. When choosing the same base of all finite unions of half cylinders $B_{i} \times\left[t_{i}, \infty\right]$ as in [25, Theorem XII-6] for random u.s.c. functions on $\mathbb{R}^{d}$, we obtain the following characterization of random marked closed sets.

Theorem 1. The distribution of a random marked closed set $(\Xi, Z)$ (as a probability measure on $\left.\Phi_{\mathrm{usc}}\right)$ is completely determined by the joint probabilities

$$
\mathrm{P}\left(\sup _{x \in B_{i} \cap \Xi} Z(x)<t_{i}, B_{i} \cap \Xi \neq \varnothing, i \in I ; B_{j} \cap \Xi=\varnothing, j \in\{1, \ldots, n\} \backslash I\right),
$$

where $B_{1}, \ldots, B_{n}$ are compact subsets of $\mathbb{R}^{d}, t_{1}, \ldots, t_{n} \in \overline{\mathbb{R}}$, and $I$ is a subset of $\{1, \ldots, n\}$, $n \in \mathbb{N}$.

Definition 2. A random marked closed set $(\Xi, Z)$ is called stationary if

$$
\mathrm{P}(\tau(\Xi, Z)+(x, 0) \in \cdot)=\mathrm{P}(\tau(\Xi, Z) \in \cdot)
$$

for all $x \in \mathbb{R}^{d}$, and it is called isotropic if

$$
\mathrm{P}(\theta \tau(\Xi, Z) \in \cdot)=\mathrm{P}(\tau(\Xi, Z) \in \cdot)
$$

for all rotations $\theta \in \mathrm{SO}_{d+1}$ with $\theta\left(\mathbb{R}^{d} \times\{0\}\right)=\mathbb{R}^{d} \times\{0\}$. 
Example 1. A particular model of a random marked closed set that describes an unbiased sampling of a random field [27] is given when $Z$ is a random u.s.c. function on $\mathbb{R}^{d}$ that is independent of the random closed set $\Xi$. We call $(\Xi, Z)$ a random field model.

If the data are consistent with a random field model, any analysis is simplified considerably since the domain and the marks can be investigated separately (see also Remark 5 below) by using standard techniques for random sets [26] and for geostatistical data [6], [10]. For the particular case of marked point processes, several tests for the random field model hypothesis have been developed [11], [24].

Example 2. Let $\Xi$ be a random closed set, and let $Z(x)=d(\partial \Xi, x)$ be the Euclidean distance of $x \in \mathbb{R}^{d}$ to the boundary of $\Xi$. Then $Z$ is continuous even on $\Xi$. Since local maxima of $Z$ are only attained at locations in the interior of $\Xi$, the random marked set $(\Xi, Z)$ is a random field model if and only if $\Xi=\partial \Xi$ almost surely, in which case $Z$ is trivial.

Example 3. Cressie et al. [7] considered the spatial prediction on a river network. Here, $\Xi$ is the flow of the river (as a one-dimensional line or a two-dimensional stripe) and $Z$ models the dissolved oxygen.

Example 4. Let $\Xi$ be a random closed set represented as a locally finite union of closed $C^{2}$-smooth hypersurfaces in $\mathbb{R}^{d}$ such that any two hypersurfaces intersect at most in a set of measure 0 with respect to the $(d-1)$-dimensional Hausdorff measure. For any $x \in \Xi$, the mark $Z(x)$ might be the maximum of the mean curvatures of the hypersurfaces at $x$. The mean curvature has its importance, for example, in the analysis of foams [15].

Remark 1. In [19] Molchanov studied labelled random closed sets in the sense that a random closed set is split into several closed subsets; see also [20, p. 141]. Here the marks are at the nominal scale. Since random marked sets link real-valued marked point processes and realvalued random fields, the concept of the present paper may be seen as an implicit generalisation of labelled random closed sets.

\section{Characteristics for random marked closed sets}

For the description of random fields, a set of second-order characteristics like the variogram, the covariance function, and the correlation function are used [6]. In analogy to these summary functions, several second-order characteristics for marked point processes have been introduced as conditional quantities given the existence of points of the respective unmarked point process [23], [26]. Since point processes can be described as random (counting) measures, these quantities have been derived as Radon-Nikodym derivatives of certain second-order moment measures [4, Section 2.7]. Nevertheless, random measures are not always appropriate for the definition of second-order characteristics as the following example illustrates.

Example 5. Let the stationary random closed set $\Xi$ in $\mathbb{R}^{1}$ be given by

$$
\Xi=\xi+\bigcup_{z \in \mathbb{Z}}[2 z-p, 2 z+p] \cup\{2 z+1\},
$$

where $p \in\left(0, \frac{1}{3}\right)$ and $\xi$ is uniformly distributed on [0,1]. Obviously, interpoint distances $r \in(0,2 p]$ are only possible if both points belong to the same segment $\xi+[2 z-p, 2 z+p]$, and interpoint distances $r \in(1-p, 1+p]$ are only possible if one point belongs to a segment $\xi+[2 z-p, 2 z+p]$ and the other is from one of the singletons, $\{\xi+2 z-1\}$ or $\{\xi+2 z+1\}$. Since $\mathrm{P}(0, r \in \Xi)=0$ for all $r \in(1-p, 1+p]$, the approach of defining second-order 
characteristics using a random measure, which is here based on the Lebesgue measure on $\mathbb{R}^{1}$, cannot account for segment-singleton point pairs, and, hence, these characteristics are undefined for $r \in(1-p, 1+p]$. Nonetheless, it does make sense to also consider the correlation of two marks given that the corresponding points are a distance $r, r \in(1-p, 1+p]$, apart.

In what follows, $B_{\varepsilon}(x)$ denotes the Euclidean ball in $\mathbb{R}^{d}$ with centre $x \in \mathbb{R}^{d}$ and radius $\varepsilon \geq 0$, ' $\oplus$ ' denotes Minkowski addition, and we use the shorthand $\Xi_{\oplus \varepsilon}$ for $\Xi \oplus B_{\varepsilon}(o)$, where $o$ is the origin in $\mathbb{R}^{d}$. Furthermore, $\mathbf{1}_{A}$ denotes the indicator of $A$.

Let $(\Xi, Z)$ be a random marked closed set in $\mathbb{R}^{d}$ with marks in $\mathbb{R}$. For ease, we assume stationarity, but the approach can be extended to a nonstationary setup. For any $\varepsilon \geq 0$, define the (stationary) random field $Z_{\varepsilon}$ by

$$
Z_{\varepsilon}(x)= \begin{cases}\max _{y \in \Xi \cap B_{\varepsilon}(x)} Z(y), & x \in \Xi_{\oplus \varepsilon}, \\ 0, & \text { otherwise. }\end{cases}
$$

Let $f: \mathbb{R}^{2} \rightarrow \mathbb{R}$ be a right-continuous function. For all $h \in \mathbb{R}^{d}$, define

$$
\kappa_{f}(h)=\lim _{\varepsilon \rightarrow 0+} \mathrm{E}\left[f\left(Z_{\varepsilon}(o), Z_{\varepsilon}(h)\right) \mid o, h \in \Xi_{\oplus \varepsilon}\right]
$$

whenever $\kappa_{|f|}(h)<\infty$ and $\mathrm{P}\left(o, h \in \Xi_{\oplus \varepsilon}\right)>0$ for all $\varepsilon>0$; otherwise, $\kappa_{f}(h)$ is undefined.

In particular, for the following choices of $f$,

$$
e\left(m_{1}, m_{2}\right)=m_{1}, \quad c\left(m_{1}, m_{2}\right)=m_{1} m_{2}, \quad \text { and } \quad v\left(m_{1}, m_{2}\right)=m_{1}^{2},
$$

define

$$
\begin{aligned}
E(h) & =\kappa_{e}(h), \\
\gamma(h) & =\frac{1}{2}\left(\kappa_{v}(h)+\kappa_{v}(-h)\right)-\kappa_{c}(h), \\
\operatorname{cov}(h) & =\kappa_{c}(h)-\kappa_{e}(h) \kappa_{e}(-h), \\
\operatorname{cor}(h) & =\frac{\kappa_{c}(h)-\kappa_{e}(h) \kappa_{e}(-h)}{\left(\kappa_{v}(h)-\kappa_{e}(h)^{2}\right)^{1 / 2}\left(\kappa_{v}(-h)-\kappa_{e}(-h)^{2}\right)^{1 / 2}}, \\
k_{m m}(h) & =(\bar{m})^{-2} \kappa_{c}(h), \quad \bar{m} \neq 0,
\end{aligned}
$$

where $\bar{m}=\mathrm{E}[Z(o) \mid o \in \Xi]$ is the mean mark.

We call $\gamma$ the mark variogram, cov the mark covariance function, cor the mark correlation function, and $k_{m m}$ Stoyan's $k_{m m}$-function of $(\Xi, Z)$ [23]. Note that, if $\Xi \equiv \mathbb{R}^{d}$, these definitions are compatible with the classical definitions for random fields (see Remark 5 below).

Whenever $(\Xi, Z)$ is assumed to be both stationary and isotropic, the characteristics given by (3)-(7) are rotation invariant. By a slight abuse of the notation we will write $E(r), r \in[0, \infty)$, instead of $E(h), h \in \mathbb{R}^{d}$. The same applies for the functions defined in (4)-(7).

Remark 2. Let $\Psi_{\varepsilon}=v_{d}\left(\cdot \cap \Xi_{\oplus \varepsilon}\right)$ be the random volume measure associated with the random closed set $\Xi_{\oplus \varepsilon}$. Here, $v_{d}$ is the $d$-dimensional Lebesgue measure. If $\mu_{\varepsilon}^{(2)}$ denotes the secondorder moment measure of $\Psi_{\varepsilon}$ then, for $B_{1}, B_{2} \in \mathcal{B}\left(\mathbb{R}^{d}\right)$, we have

$$
\begin{array}{r}
\int_{B_{2}} \int_{B_{1}} \mathrm{E}\left[f\left(Z_{\varepsilon}(x), Z_{\varepsilon}(y)\right) \mathbf{1}_{\Xi_{\oplus \varepsilon}}(x) \mathbf{1}_{\Xi_{\oplus \varepsilon}}(y)\right] \mathrm{d} x \mathrm{~d} y \\
=\mathrm{E}\left[\int_{B_{2}} \int_{B_{1}} f\left(Z_{\varepsilon}(x), Z_{\varepsilon}(y)\right) \Psi_{\varepsilon}(\mathrm{d} x) \Psi_{\varepsilon}(\mathrm{d} y)\right]
\end{array}
$$




$$
\begin{aligned}
& =\int_{B_{1} \times B_{2}} \int_{\mathbb{R}^{2}} f\left(m_{1}, m_{2}\right) Q_{\varepsilon ; x, y}\left(\mathrm{~d}\left(m_{1}, m_{2}\right)\right) \mu_{\varepsilon}^{(2)}(\mathrm{d}(x, y)) \\
& =\int_{B_{2}} \int_{B_{1}} \int_{\mathbb{R}^{2}} f\left(m_{1}, m_{2}\right) Q_{\varepsilon ; x, y}\left(\mathrm{~d}\left(m_{1}, m_{2}\right)\right) \mathrm{P}\left(x, y \in \Xi_{\oplus \varepsilon}\right) \mathrm{d} x \mathrm{~d} y,
\end{aligned}
$$

where $Q_{\varepsilon ; x, y}$ is the two-point mark distribution of the weighted random measure $\left(\Psi_{\varepsilon}, Z_{\varepsilon}\right)$ [4]. Hence, for almost all $(x, y)$ with $\mathrm{P}\left(x, y \in \Xi_{\oplus \varepsilon}\right)>0$, we have

$$
\mathrm{E}\left[f\left(Z_{\varepsilon}(x), Z_{\varepsilon}(y)\right) \mid x, y \in \Xi_{\oplus \varepsilon}\right]=\int_{\mathbb{R}^{2}} f\left(m_{1}, m_{2}\right) Q_{\varepsilon ; x, y}\left(\mathrm{~d}\left(m_{1}, m_{2}\right)\right) .
$$

Remark 3. In the case $\mathrm{P}(o, h \in \Xi)>0, h \in \mathbb{R}^{d}$, the above definition takes the simpler form

$$
\kappa_{f}(h)=\mathrm{E}[f(Z(o), Z(h)) \mid o, h \in \Xi]
$$

for $f \in\{e, c, v\}$ if we impose the integrability conditions

$$
\begin{gathered}
\mathrm{E}\left[|Z(o)|^{2} \mathbf{1}_{\Xi}(o)\right]<\infty, \quad \kappa_{v}(h)<\infty, \\
\text { and } \quad \lim _{\varepsilon \rightarrow 0+} \mathrm{E}\left[\left(Z(p(\Xi, o))_{-}\right)^{2} \mathbf{1}_{\Xi_{\oplus \varepsilon} \backslash \Xi}(o)\right]<\infty .
\end{gathered}
$$

Here $e, c$, and $v$ are given by (2), $a_{-}$denotes the negative part of $a \in \mathbb{R}$, and $p(A, x)$ is the metric projection [12] of $x \in \mathbb{R}^{d} \backslash \Xi$ onto the boundary $\partial \Xi$ of $\Xi$ with the smallest coordinates lexicographically ordered, say. Note that the latter is not crucial, since, due to stationarity, the probability that the projection of $o$ onto $\partial \Xi$ is not unique is 0 ; see [12].

Equality (8) can be seen as follows. Denoting by $a_{+}$the positive part of $a \in \mathbb{R}$ we always have

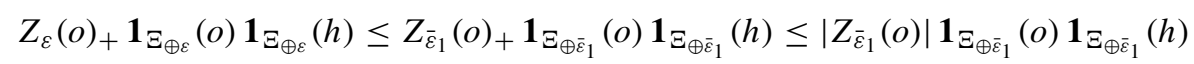

for all $0<\varepsilon \leq \bar{\varepsilon}_{1}$, where the right-hand side is integrable for small enough $\bar{\varepsilon}_{1}$ as $\kappa_{|e|}(h)<\infty$. Similarly,

$$
Z_{\varepsilon}(o)_{-} \mathbf{1}_{\Xi}(o) \mathbf{1}_{\Xi_{\oplus \varepsilon}}(h) \leq Z_{\varepsilon}(o)_{-} \mathbf{1}_{\Xi}(o) \leq Z(o)_{-} \mathbf{1}_{\Xi}(o) \leq|Z(o)| \mathbf{1}_{\Xi}(o) .
$$

Finally, since $Z_{\varepsilon}(o)_{-}=\min _{x \in \Xi \cap B_{\varepsilon}(o)} Z(x)_{-}$for $o \in \Xi_{\oplus \varepsilon} \backslash \Xi$, we have

$$
\begin{aligned}
Z_{\varepsilon}(o)_{-} \mathbf{1}_{\Xi_{\oplus \varepsilon} \backslash \Xi}(o) \mathbf{1}_{\Xi_{\oplus \varepsilon}}(h) & \leq Z_{\varepsilon}(o)_{-} \mathbf{1}_{\Xi_{\oplus \varepsilon} \backslash \Xi}(o) \\
& \leq Z(p(\Xi, o))_{-} \mathbf{1}_{\Xi_{\oplus \varepsilon} \backslash \Xi}(o) \\
& \leq Z(p(\Xi, o))_{-} \mathbf{1}_{\Xi_{\oplus \bar{\varepsilon}_{2}}}(o)
\end{aligned}
$$

for all $0<\varepsilon \leq \bar{\varepsilon}_{2}$, where the right-hand side is integrable for small enough $\bar{\varepsilon}_{2}$. Note that

$$
\mathrm{E}\left[|Z(o)| \mathbf{1}_{\Xi}(o)\right]<\infty, \quad \kappa_{|e|}(h)<\infty, \quad \lim _{\varepsilon \rightarrow 0+} \mathrm{E}\left[Z(p(\Xi, o))_{-} \mathbf{1}_{\Xi_{\oplus \varepsilon} \backslash \Xi}(o)\right]<\infty
$$

by the Cauchy-Schwarz inequality. Similarly, we find that

$$
\left|Z_{\bar{\varepsilon}_{1}}(o)\right|^{2} \mathbf{1}_{\Xi_{\oplus \bar{\varepsilon}_{1}}}(o) \mathbf{1}_{\Xi_{\oplus \bar{\varepsilon}_{1}}}(h)+|Z(o)|^{2} \mathbf{1}_{\Xi}(o)+\left(Z(p(\Xi, o))_{-}\right)^{2} \mathbf{1}_{\Xi_{\oplus \bar{\varepsilon}_{2}}}(o)
$$

is an integrable upper bound of $\left|Z_{\varepsilon}(o)\right|^{2} \mathbf{1}_{\Xi_{\oplus \varepsilon}}(o) \mathbf{1}_{\Xi_{\oplus \varepsilon}}(h)$ and is half of the integrable upper bound of $\left|Z_{\varepsilon}(o) Z_{\varepsilon}(h)\right| \mathbf{1}_{\Xi_{\oplus \varepsilon}}(o) \mathbf{1}_{\Xi_{\oplus \varepsilon}}(h)$ due to the estimate $\left|Z_{\varepsilon}(o) Z_{\varepsilon}(h)\right| \leq \frac{1}{2}\left(\left|Z_{\varepsilon}(o)\right|^{2}+\right.$ $\left.\left|Z_{\varepsilon}(h)\right|^{2}\right)$. 
Since $Z$ is u.s.c. on $\Xi$, a value $\varepsilon>0$ exists for every $x \in \Xi$ and every $\delta>0$ such that $Z(y) \leq Z(x)+\delta$ for all $y \in B_{\varepsilon}(x) \cap \Xi$. Hence, we have $Z_{\varepsilon}(x) \rightarrow Z(x)$ from above as $\varepsilon \rightarrow 0+$. Furthermore, $x \notin \Xi$ implies that $x \notin \Xi_{\oplus \varepsilon}$ for all sufficiently small $\varepsilon$. We then have

$$
f\left(Z_{\varepsilon}(o), Z_{\varepsilon}(h)\right) \mathbf{1}_{\mathbf{\Xi}_{\oplus \varepsilon}}(o) \mathbf{1}_{\mathbf{\Xi}_{\oplus \varepsilon}}(h) \rightarrow f(Z(o), Z(h)) \mathbf{1}_{\Xi}(o) \mathbf{1}_{\Xi}(h) \text { almost surely }
$$

as $\varepsilon \rightarrow 0+$. Hence, by the dominated convergence theorem we have

$$
\kappa_{f}(h)=\lim _{\varepsilon \rightarrow 0+} \frac{\mathrm{E}\left[f\left(Z_{\varepsilon}(o), Z_{\varepsilon}(h)\right) \mathbf{1}_{\Xi_{\oplus \varepsilon}}(o) \mathbf{1}_{\Xi_{\oplus \varepsilon}}(h)\right]}{\mathrm{P}\left(o, h \in \Xi_{\oplus \varepsilon}\right)}=\frac{\mathrm{E}\left[f(Z(o), Z(h)) \mathbf{1}_{\Xi}(o) \mathbf{1}_{\Xi}(h)\right]}{\mathrm{P}(o, h \in \Xi)} .
$$

Remark 4. There exists an alternative concept of random marked sets inspired by the notion of random fields that allows second-order characteristics in the sense of the preceding remark to be defined.

Let $\overline{\mathbb{R}} \varnothing=\overline{\mathbb{R}} \cup\left\{\zeta_{\varnothing}\right\}$ be the extension of $\overline{\mathbb{R}}$ by some $\zeta \varnothing$. We denote by $\mathcal{B}(\overline{\mathbb{R}} \varnothing)$ the respective Borel $\sigma$-field which is generated by all sets $B_{1} \cup B_{2}$ for $B_{1} \in \mathscr{B}(\mathbb{R})$ and $B_{2} \subset\{-\infty, \infty, \zeta \varnothing\}$.

A family of random variables $Z(\cdot, x): \Omega \rightarrow \overline{\mathbb{R}}_{\varnothing}, x \in \mathbb{R}^{d}$, on the probability space $(\Omega, \mathcal{A}, \mathrm{P})$ is called a random field with random domain $\Xi$ if

$$
\Xi=\left\{x \in \mathbb{R}^{d}: Z(\cdot, x) \neq \zeta_{\varnothing}\right\} .
$$

Clearly, when $Z$ only takes values different from $\zeta_{\varnothing}$ or $\left\{-\infty, \infty, \zeta_{\varnothing}\right\}$, this notion of a random marked set includes the usual $\overline{\mathbb{R}}$ - or $\mathbb{R}$-valued random field on $\mathbb{R}^{d}$.

Note that $\Xi$ is a random set in a very general sense [18, pp. 40-45], entirely determined by its indicator $\mathbf{1}_{\Xi}(x)=\mathbf{1}_{\overline{\mathbb{R}}}(Z(x))$. If $Z$ is jointly measurable, i.e. $Z$ is $\left(\mathcal{A} \otimes \mathscr{B}\left(\mathbb{R}^{d}\right), \mathscr{B}\left(\overline{\mathbb{R}}_{\varnothing}\right)\right)$ measurable, then the realizations of $\Xi$ are almost surely Borel measurable. If we additionally have almost surely closed (open) realizations of $\Xi$ then $Z$ is called a random field with random closed (open) domain; see also [20].

If $\mathrm{P}(o \in \Xi)>0$ holds for a stationary random field $Z$ with random domain $\Xi$, we can define second-order characteristics without any further assumption on path regularity. Let $\tilde{Z}$ be the (stationary) random field given by $\tilde{Z}(x)=Z(x)$ for $x \in \Xi$, and $\tilde{Z}(x)=0$ otherwise. Let $f: \mathbb{R}^{2} \rightarrow \mathbb{R}$ be a measurable function. For all $h \in \mathbb{R}^{d}$, define

$$
\kappa_{f}(h)=\mathrm{E}[f(\tilde{Z}(o), \tilde{Z}(h)) \mid o, h \in \Xi]
$$

whenever $\mathrm{P}(o, h \in \Xi)>0$ and $\mathrm{E}\left[|f(\tilde{Z}(o), \tilde{Z}(h))| \mathbf{1}_{\Xi}(o) \mathbf{1}_{\Xi}(h)\right]<\infty$.

Remark 5. Let $(\Xi, Z)$ be a stationary real-valued random-field model, and let

$$
Z_{\varepsilon}(x)= \begin{cases}\max _{y \in \Xi \cap B_{\varepsilon}(x)} Z(y), & x \in \Xi_{\oplus \varepsilon} \\ Z(x), & \text { otherwise. }\end{cases}
$$

Since $Z$ is u.s.c. on $\Xi$, we have $Z_{\varepsilon}(x) \rightarrow Z(x)$ from above for $x \in \Xi$, and, hence, by the definition of $Z_{\varepsilon}$, for all $x \in \mathbb{R}^{d}$ as $\varepsilon \rightarrow 0+$. Then, using the independence of $Z$ and $\Xi$, we obtain

$$
\begin{aligned}
\kappa_{f}(h) & =\lim _{\varepsilon \rightarrow 0+} \mathrm{E}\left[f\left(Z_{\varepsilon}(o), Z_{\varepsilon}(h)\right) \mid o, h \in \Xi_{\oplus \varepsilon}\right] \\
& =\lim _{\varepsilon \rightarrow 0+} \mathrm{E}\left[f\left(Z_{\varepsilon}(o), Z_{\varepsilon}(h)\right)\right] \\
& =\mathrm{E}[f(Z(o), Z(h))]
\end{aligned}
$$

for all $h \in \mathbb{R}^{d}$ which satisfy $\mathrm{P}\left(o, h \in \Xi_{\oplus \varepsilon}\right)>0$ for all $\varepsilon>0$ and, depending on the choice of $f$ according to (2), one of the integrability conditions in Remark 3 with $\Xi$ replaced by $\mathbb{R}^{d}$. 
Remark 6. The definition of $\kappa_{f}$ according to (1) is, in important situations, consistent with the classical definition of the second-order characteristics of stationary marked point processes [23]. Let $\widetilde{\Phi}$ be a stationary simple marked point process on $\mathbb{R}^{d} \times \mathbb{R}$. Then $\Xi$ is the support of the unmarked point process $\Phi=\widetilde{\Phi}(\cdot \times \mathbb{R})$. We assume that the second-order moment measure $\mu^{(2)}$ of $\Phi$ is locally finite. Denoting by $\mathbf{1}_{\{\cdot\}}$ the indicator of the event $\{\cdot\}$, we have

$$
\begin{aligned}
\mathrm{E}\left[f\left(Z_{\varepsilon}(o), Z_{\varepsilon}(h)\right) \mathbf{1}_{\Xi \oplus B_{\varepsilon}(o)}(o) \mathbf{1}_{\Xi \oplus B_{\varepsilon}(o)}(h)\right] \\
=\mathrm{E}\left[f\left(Z_{\varepsilon}(o), Z_{\varepsilon}(h)\right) \mathbf{1}_{\left\{\Phi\left(B_{\varepsilon}(o)\right)=1\right\}} \mathbf{1}_{\left\{\Phi\left(B_{\varepsilon}(h)\right)=1\right\}}\right] \\
\quad+\mathrm{E}\left[f\left(Z_{\varepsilon}(o), Z_{\varepsilon}(h)\right) \mathbf{1}_{\left\{\Phi\left(B_{\varepsilon}(o)\right)>1\right\}} \mathbf{1}_{\left\{\Phi\left(B_{\varepsilon}(h)\right) \geq 1\right\}}\right] \\
\quad+\mathrm{E}\left[f\left(Z_{\varepsilon}(o), Z_{\varepsilon}(h)\right) \mathbf{1}_{\left\{\Phi\left(B_{\varepsilon}(o)\right)=1\right\}} \mathbf{1}_{\left\{\Phi\left(B_{\varepsilon}(h)\right)>1\right\}}\right]
\end{aligned}
$$

for $\|h\|>0$. For any $0<\varepsilon<\|h\| / 2$, the first summand equals

$$
\mathrm{E}\left[\sum_{\left(x_{1}, m_{1}\right),\left(x_{2}, m_{2}\right) \in \widetilde{\Phi}} f\left(m_{1}, m_{2}\right) \mathbf{1}_{B_{\varepsilon}(o)}\left(x_{1}\right) \mathbf{1}_{B_{\varepsilon}(h)}\left(x_{2}\right)\right]=: \mu_{f}^{(2)}\left(B_{\varepsilon}(o) \times B_{\varepsilon}(h)\right) .
$$

We can extend the argumentation in [8, Proposition 9.3.XV] in order to conclude that

$$
\frac{\mathrm{P}\left(o, h \in \Xi_{\oplus \varepsilon}\right)}{\mu^{(2)}\left(B_{\varepsilon}(o) \times B_{\varepsilon}(h)\right)}=\frac{\mathrm{P}\left(\Phi\left(B_{\varepsilon}(o)\right) \geq 1, \Phi\left(B_{\varepsilon}(h)\right) \geq 1\right)}{\mu^{(2)}\left(B_{\varepsilon}(o) \times B_{\varepsilon}(h)\right)} \rightarrow 1
$$

as $\varepsilon \rightarrow 0+$. If we additionally impose the condition that, for some $\bar{\varepsilon}>0$,

$$
\sup _{\varepsilon \in(0, \bar{\varepsilon})} \frac{\mathrm{E}\left[\left|f\left(Z_{\varepsilon}(o), Z_{\varepsilon}(h)\right)\right| \mathbf{1}_{\left\{\Phi\left(B_{\varepsilon}(o)\right)>1\right\}} \mathbf{1}_{\left\{\Phi\left(B_{\varepsilon}(h)\right) \geq 1\right\}} \mathbf{1}_{\left\{\left|f\left(Z_{\varepsilon}(o), Z_{\varepsilon}(h)\right)\right|>M\right\}}\right]}{\mathrm{P}\left(\Phi\left(B_{\varepsilon}(o)\right) \geq 1, \Phi\left(B_{\varepsilon}(h)\right) \geq 1\right)} \rightarrow 0
$$

as $M \rightarrow \infty$, we obtain

$$
\kappa_{f}(h)=\lim _{\varepsilon \rightarrow 0+} \frac{\mu_{f}^{(2)}\left(B_{\varepsilon}(o) \times B_{\varepsilon}(h)\right)}{\mu^{(2)}\left(B_{\varepsilon}(o) \times B_{\varepsilon}(h)\right)},
$$

which equals $\mu^{(2)}$-almost everywhere the Radon-Nikodym derivative

$$
\frac{\mathrm{d} \mu_{f}^{(2)}(x, x+h)}{\mathrm{d} \mu^{(2)}(x, x+h)}
$$

For instance, the above condition is satisfied if $\mathrm{E}\left|f\left(Z_{\varepsilon}(o), Z_{\varepsilon}(h)\right)\right|^{\alpha}$ is uniformly bounded on $(0, \bar{\varepsilon})$ for some $\alpha>1$.

A function $f: \mathbb{R}^{d} \rightarrow \mathbb{R}$ is called positive definite if

$$
\sum_{i=1}^{n} \sum_{j=1}^{n} a_{i} a_{j} f\left(x_{i}-x_{j}\right) \geq 0
$$

for any $n \in \mathbb{N}, x_{1}, \ldots, x_{n} \in \mathbb{R}^{d}$, and $a_{1}, \ldots, a_{n} \in \mathbb{R}$, and it is called conditionally negative definite if

$$
\sum_{i=1}^{n} \sum_{j=1}^{n} a_{i} a_{j} f\left(x_{i}-x_{j}\right) \leq 0
$$

for any $n \in \mathbb{N}, x_{1}, \ldots, x_{n} \in \mathbb{R}^{d}$, and all $a_{1}, \ldots, a_{n} \in \mathbb{R}$ with $\sum_{i=1}^{n} a_{i}=0$. 
For a random field model, all second-order characteristics coincide with those of a random field with u.s.c. paths (see Remark 5), and, thus, share the same definiteness properties. On the other hand, for marked point processes, it has been shown through examples [28] and systematically [23] that the mark covariance function, the mark correlation function, and the $k_{m m}$-function need not be positive definite, and that the mark variogram need not be conditionally negative definite in contrast to random fields. Some of the constructions used in [23] are based on the fact that, for a marked point process, $\Xi$ is a locally finite subset of $\mathbb{R}^{d}$ and has therefore Lebesgue measure 0. However, the next example shows that in general we cannot expect the mark covariance function to be positive definite (or the mark correlation function and $k_{m m}$-function) even when we have $\mathrm{E}\left[v_{d}\left(\Xi \cap[0,1]^{d}\right)\right]=\mathrm{P}(o \in \Xi)>0$.

Example 6. (Example 2 continued.) Let $p \in\left(\frac{2}{3}, 1\right], \xi$ be a random variable uniformly distributed on $[0,1], \Xi=\mathbb{Z} \oplus[\xi, p+\xi]$, and $Z(\xi, \cdot)$ be a 1 -periodic function defined by

$$
Z(\xi, x)= \begin{cases}x-\xi, & x \in \mathbb{Z} \oplus\left[\xi, \frac{1}{2} p+\xi\right), \\ p-(x-\xi), & x \in \mathbb{Z} \oplus\left[\frac{1}{2} p+\xi, p+\xi\right), \\ 0, & x \in \mathbb{Z} \oplus[p+\xi, 1+\xi) .\end{cases}
$$

Then $Z$ and $\Xi$ are jointly stationary and each of the characteristics given by (3)-(7) is 1-periodic. In particular, on $\left[0, \frac{1}{2}\right.$ ) we have

$$
\operatorname{cov}(r)= \begin{cases}\frac{p^{4}-4 p^{3} r-12 p^{2} r^{2}+48 p r^{3}-36 r^{4}}{48(p-r)^{2}}, & r \in[0,1-p), \\ \frac{32 r^{3}(2 p-1)+24 r(1-r)-48 p r(1-p)(1-r)-12 p^{2}(2 p r-p+1)-3 p^{4}+8 p-4}{48(2 p-1)^{2}}, & r \in\left[1-p, \frac{1}{2} p\right), \\ -\frac{4 p^{4}-8 p^{3}+6 p^{2}-2 p+12 r^{4}-24 r^{3}+18 r^{2}-6 r+1}{12(2 p-1)^{2}}, & r \in\left[\frac{1}{2} p, \frac{1}{2}\right],\end{cases}
$$

and, by symmetry, $\operatorname{cov}(r)=\operatorname{cov}(1-r)$ for $r \in\left(\frac{1}{2}, 1\right)$. Since cov is 1 -periodic the 0 th coefficient of the Fourier series of cov is proportional to

$$
\int_{0}^{1} \operatorname{cov}(r) \mathrm{d} r=\frac{7}{6} p^{3} \ln \left(\frac{p}{2 p-1}\right)+\frac{409 p^{5}-790 p^{4}+565 p^{3}-280 p^{2}+120 p-24}{120(2 p-1)^{2}},
$$

which is negative for $\frac{2}{3} \leq p<1$ (and vanishes for $p=1$, which is the random field case). Since cov is continuous, Bochner's theorem [22] implies that cov cannot be a positive definite function.

In the next section we concentrate on our major example since some results might be of interest not only to the field of random marked sets but also to the theory of positive definite functions.

\section{Gaussian random fields exceeding $t \in \mathbb{R}$}

Let $Z$ be a stationary and isotropic centred unit variance Gaussian random field in $\mathbb{R}^{d}$. Then, for $t \in \mathbb{R}$, we define

$$
\Xi_{t}=\left\{x \in \mathbb{R}^{d}: Z(x) \geq t\right\} .
$$

If, in particular, $Z$ is almost surely continuous [1], [2] then $\Xi_{t}$ is almost surely closed, i.e. $\left(\Xi_{t}, Z\right)$ is a random marked closed set. Note that $\Xi_{t}$ is a so-called excursion set which has been extensively studied in the literature; see [1], [2], and the references therein. 
Since $Z$ is assumed to be both stationary and isotropic, its covariance function, $\operatorname{cov}(x, y)=$ $\mathrm{E}[Z(x) Z(y)], x, y \in \mathbb{R}^{d}$, is translation and rotation invariant, i.e. there exists a function $R:[0, \infty) \rightarrow \mathbb{R}$ such that $\operatorname{cov}(x, y)=R(\|x-y\|)$ for $x, y \in \mathbb{R}^{d}$.

First, we consider the case $t=0$.

Theorem 2. Let $Z$ be a stationary and isotropic centred unit variance Gaussian random field in $\mathbb{R}^{d}$ with covariance function given by $R:[0, \infty) \rightarrow \mathbb{R}$. Then, for $r \in[0, \infty)$, the second-order characteristics of $\left(\Xi_{0}, Z\right)$ are given by

$$
\begin{aligned}
E(r) & =\sqrt{\frac{\pi}{2}} \frac{1+R(r)}{\arcsin (R(r))+\pi / 2}, \\
\operatorname{cov}(r) & =R(r)+\frac{\sqrt{1-R(r)^{2}}}{\arcsin (R(r))+\pi / 2}-\frac{\pi}{2} \frac{(1+R(r))^{2}}{(\arcsin (R(r))+\pi / 2)^{2}}, \\
\gamma(r) & =(1-R(r))\left(1-\frac{\sqrt{1-R(r)^{2}}}{\arcsin (R(r))+\pi / 2}\right), \\
k_{m m}(r) & =\frac{\pi}{2}\left(R(r)+\frac{\sqrt{1-R(r)^{2}}}{\arcsin (R(r))+\pi / 2}\right), \\
\operatorname{cor}(r) & =\frac{R(r)(\arcsin (R(r))+\pi / 2)^{2}+\sqrt{1-R(r)^{2}}(\arcsin (R(r))+\pi / 2)-\pi(1+R(r))^{2} / 2}{(\arcsin (R(r))+\pi / 2)^{2}+R(r) \sqrt{1-R(r)^{2}}(\arcsin (R(r))+\pi / 2)-\pi(1+R(r))^{2} / 2} .
\end{aligned}
$$

Obviously, each of the second-order characteristics of $\left(\Xi_{0}, Z\right)$ is a continuous transform of $R$. In particular, this means that the continuity of $R$ is preserved, and, due to the monotonicity of the transform for cov (see Theorem 3 below), the curve progression of cov indicates whether or not $R$ is continuous at the origin [6].

Since, for every stationary random field model, $E(h), h \in \mathbb{R}^{d}$, is constant, (9) implies that $\left(\Xi_{0}, Z\right)$ is not a random field model, i.e. there does not exist a random field in $\mathbb{R}^{d}$ whose secondorder characteristics coincide with those of $\left(\Xi_{0}, Z\right)$ unless $R$ is constant. This is underlined by the fact that the $k_{m m}$-function of $\left(\Xi_{0}, Z\right)$ is not positive definite, in general.

Proposition 2. Let $Z$ be a stationary and isotropic centred unit variance Gaussian random field in $\mathbb{R}^{d}$ with covariance function given by a continuous function $R:[0, \infty) \rightarrow \mathbb{R}$. Then the $k_{m m}$-function of $\left(\Xi_{0}, Z\right)$ is positive definite if and only if $R \equiv 1$.

Proof. Let $q(\rho)=\rho+\sqrt{1-\rho^{2}}(\arcsin (\rho)+\pi / 2)^{-1}$. Then $q(1)=1$ and

$$
q(\rho) \geq \rho+\pi^{-1} \sqrt{1-\rho^{2}}>1, \quad \rho \in\left(\left(\pi^{2}-1\right) /\left(\pi^{2}+1\right), 1\right) .
$$

Hence, $k_{m m}(r)$ is not a positive definite function if $R \not \equiv 1$ [22, Theorem 1.4.1].

Furthermore, the mark variogram of $\left(\Xi_{0}, Z\right)$ is in general not conditionally negative definite, which can be seen as follows. Consider $\left(\Xi_{0}, Z\right)$ for dimension $d=1$ and $R(r)=\cos (r)$. Since $\gamma$ is conditionally negative definite if and only if $\mathrm{e}^{-s \gamma}$ is positive definite for all $s>0$ [22, Theorem 6.1.9], it suffices to show that $\mathrm{e}^{-\gamma}$ is not positive definite. Observe that $\mathrm{e}^{-\gamma(r)}$ inherits $2 \pi$-periodicity from $\cos (r)$, and, hence, it is positive definite if and only if its Fourier coefficients are nonnegative. Numerical calculations show that the first Fourier coefficient is near -0.03364 .

It is quite surprising to see that we are not able to falsify the claim that $\left(\Xi_{0}, Z\right)$ is a random field model by using the mark covariance function or the mark correlation function of $\left(\Xi_{0}, Z\right)$. 
Theorem 3. The functions $f_{0}:[-1,1] \rightarrow \mathbb{R}$ defined by

$$
f_{0}(\rho)=\rho+\frac{\sqrt{1-\rho^{2}}}{\arcsin \rho+\pi / 2}-\frac{\pi}{2} \frac{(1+\rho)^{2}}{(\arcsin \rho+\pi / 2)^{2}}
$$

and $g_{0}:[-1,1] \rightarrow \mathbb{R}$ defined by

$$
g_{0}(\rho)=\frac{\rho(\arcsin \rho+\pi / 2)^{2}+\sqrt{1-\rho^{2}}(\arcsin \rho+\pi / 2)-\pi(1+\rho)^{2} / 2}{(\arcsin \rho+\pi / 2)^{2}+\rho \sqrt{1-\rho^{2}}(\arcsin \rho+\pi / 2)-\pi(1+\rho)^{2} / 2}
$$

are absolutely monotone on $[0,1]$, i.e. they have only nonnegative derivatives there.

See [3] for a proof of Theorem 3.

Corollary 1. $\operatorname{cov}(r)$ and $\operatorname{cor}(r)$ are positive definite functions.

Now consider the more general case $t \in \mathbb{R}$. Unfortunately, unlike the case $t=0$, we cannot express all the second-order characteristics of $\left(\Xi_{t}, Z\right)$ in closed form. In particular, for a stationary and isotropic centred unit variance Gaussian random field $Z$ in $\mathbb{R}^{d}$ with covariance function given by $R:[0, \infty) \rightarrow \mathbb{R}$, we have

$$
\mathrm{P}\left(o, h \in \Xi_{t}\right)=\int_{0}^{R(\|h\|)} \varphi(t, t, s) \mathrm{d} s+\Psi(t)^{2} ;
$$

see [5, Equation (10.8.3)]. Here,

$$
\Psi(t)=\int_{t}^{\infty} \varphi(s) \mathrm{d} s, \quad \varphi(t)=\frac{1}{\sqrt{2 \pi}} \mathrm{e}^{-t^{2} / 2}, \quad t \in \mathbb{R},
$$

denotes the tail probability function of the standard Gaussian distribution. By $\varphi(x, y, \rho)$ we denote the density of the bivariate Gaussian distribution with unit variances and correlation $\rho$. In the following we concentrate on the mark covariance function and the mark variogram of $\left(\Xi_{t}, Z\right)$, and write

$$
P_{t}(\rho)=\int_{0}^{\rho} \varphi(t, t, s) \mathrm{d} s+\Psi(t)^{2}, \quad \rho \in[-1,1] .
$$

Lemma 1. Let $Z$ be a stationary and isotropic centred unit variance Gaussian random field in $\mathbb{R}^{d}$ with covariance function given by $R:[0, \infty) \rightarrow \mathbb{R}$. Then, for $t \in \mathbb{R}$ and $h \in \mathbb{R}^{d}$, we have

$$
\begin{gathered}
\mathrm{E}\left[Z(o) \mathbf{1}_{\Xi_{t}}(o) \mathbf{1}_{\Xi_{t}}(h)\right]=E_{t}(R(\|h\|)), \\
E_{t}(\rho)=\varphi(t)(\rho+1) \Psi\left(t \sqrt{\frac{1-\rho}{1+\rho}}\right), \\
\mathrm{E}\left[Z(o) Z(h) \mathbf{1}_{\Xi_{t}}(o) \mathbf{1}_{\Xi_{t}}(h)\right]=C_{t}(R(\|h\|)),
\end{gathered}
$$

where

$$
\begin{gathered}
C_{t}(\rho)=\left(1-\rho^{2}\right) \varphi(t, t, s)+2 \rho t \varphi(t) \Psi\left(t \sqrt{\frac{1-\rho}{1+\rho}}\right)+\rho P_{t}(\rho), \\
\mathrm{E}\left[Z(o)^{2} \mathbf{1}_{\Xi_{t}}(o) \mathbf{1}_{\Xi_{t}}(h)\right]=V_{t}(R(\|h\|)),
\end{gathered}
$$

and

$$
V_{t}(\rho)=\rho\left(1-\rho^{2}\right) \varphi(t, t, s)+\left(1+\rho^{2}\right) t \varphi(t) \Psi\left(t \sqrt{\frac{1-\rho}{1+\rho}}\right)+P_{t}(\rho)
$$



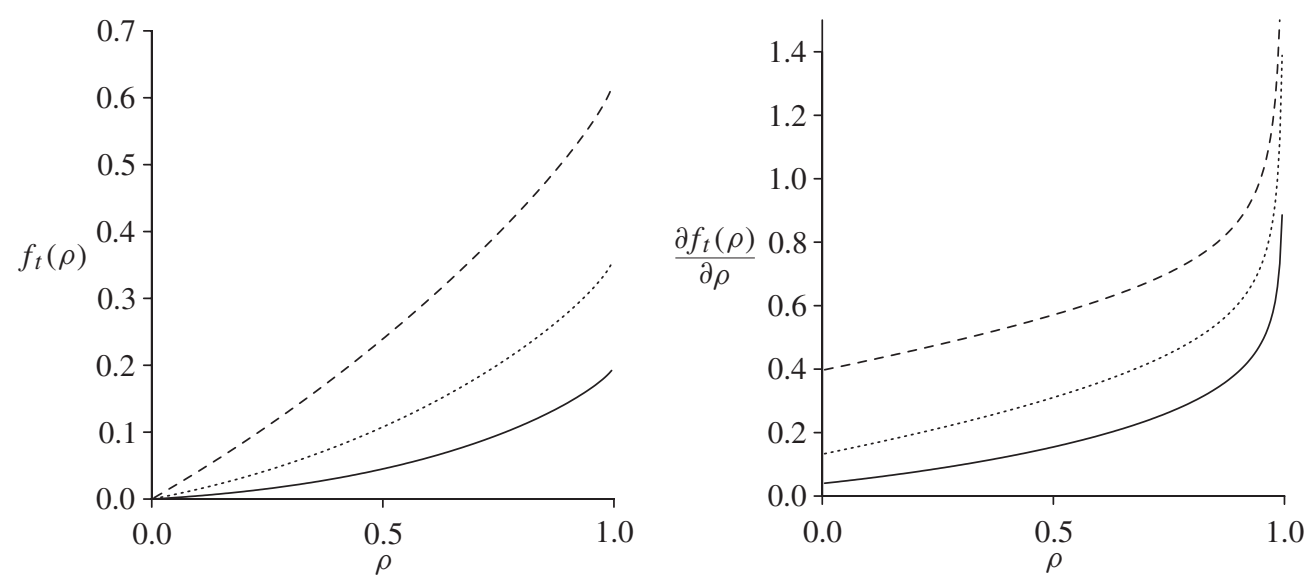

FiguRe 1: $f_{t}$ (left) and $f_{t}^{\prime}$ (right) for $t=-1$ (dashed lines), $t=0$ (dotted lines), and $t=1$ (solid lines).

Then the mark covariance function of $\left(\Xi_{t}, Z\right)$ is given by

$$
\operatorname{cov}(r)=f_{t}(R(r)), \quad f_{t}(\rho)=\frac{C_{t}(\rho)}{P_{t}(\rho)}-\frac{E_{t}(\rho)^{2}}{P_{t}(\rho)^{2}},
$$

and the mark variogram of $\left(\Xi_{t}, Z\right)$ is given by

$$
\gamma(r)=v_{t}(R(r)), \quad v_{t}(\rho)=\frac{V_{t}(\rho)-C_{t}(\rho)}{P_{t}(\rho)} .
$$

There is strong evidence that the mark covariance function of $\left(\Xi_{t}, Z\right), t \neq 0$, is also positive definite for a certain class of Gaussian random fields $Z$. In Figure 1 we plot $f_{t}(\rho)$ and $f_{t}^{\prime}(\rho)$ for several $t$, and see that, for these $t$, the functions $f_{t}(\rho)$ are both increasing and convex for $\rho \in[0,1]$. Hence, if this is really true then Pólya's criterion [21] for instance would imply that, for any continuous and convex function $R:[0, \infty) \rightarrow \mathbb{R}$ satisfying $R(0)=1$ and $\lim _{r \rightarrow \infty} R(r)=0$, the function $f_{t}(R(|\cdot|))$ is positive definite on $\mathbb{R}$.

Remark 7. The mark covariance function $\operatorname{cov}(r)=f_{t}(R(r))$ of $\left(\Xi_{t}, Z\right)$ has a right-hand derivative at 0 which does not vanish. Let $Z$ be a stationary and isotropic centred unit variance Gaussian random field in $\mathbb{R}^{d}$ with continuous covariance function $R:[0, \infty) \rightarrow \mathbb{R}$, excluding $R \equiv 1$. Then

$$
\operatorname{cov}^{\prime}(0+)=\frac{\left(t^{2}-1\right) \Psi(t)^{2}-3 t \varphi(t) \Psi(t)+2 \varphi(t)^{2}}{\Psi(t)^{3}} C_{\Xi_{t}}^{\prime}(0+)<0 .
$$

Here, $C_{\Xi_{t}}(\|h\|)=\mathrm{P}\left(o, h \in \Xi_{t}\right)$ is the set covariance of the excursion set $\Xi_{t}$, and we have

$$
C_{\Xi_{t}}^{\prime}(0+)=-\frac{\varphi(t)}{\sqrt{2 \pi}} \sqrt{-R^{\prime \prime}(0+)}<0
$$

when $Z$ is mean-square differentiable, and $\operatorname{cov}^{\prime}(0+)=-\infty$ otherwise. In either case, the right-hand derivative of the mark variogram $\gamma(r)=v_{t}(R(r))$ of $\left(\Xi_{t}, Z\right)$ at $r=0$ is $-R^{\prime}(0+)$. See [3] for a proof. 


\section{Proofs}

First, we prove Lemma 1 of Section 4 which is needed in the proof of Theorem 2.

\subsection{Proof of Lemma 1}

We will repeatedly apply the identities

$$
\begin{gathered}
\varphi^{\prime}(y)=-y \varphi(y), \\
\varphi(y, x, \rho)=\varphi(x, y, \rho)=\frac{1}{\sqrt{1-\rho^{2}}} \varphi(x) \varphi\left(\frac{y-\rho x}{\sqrt{1-\rho^{2}}}\right), \\
\int_{t}^{\infty} \frac{1}{\sqrt{1-\rho^{2}}} \varphi\left(\frac{t-\rho x}{\sqrt{1-\rho^{2}}}\right) \varphi(x) \mathrm{d} x=\varphi(t) \Psi\left(t \sqrt{\frac{1-\rho}{1+\rho}}\right) .
\end{gathered}
$$

By (11), the change of variable $x_{1}=\sqrt{1-\rho^{2}} y+\rho x_{2},(10)$, and (12), we have

$$
\begin{aligned}
E_{t}(\rho) & =\int_{t}^{\infty} \int_{t}^{\infty} x_{1} \frac{1}{\sqrt{1-\rho^{2}}} \varphi\left(\frac{x_{1}-\rho x_{2}}{\sqrt{1-\rho^{2}}}\right) \varphi\left(x_{2}\right) \mathrm{d} x_{1} \mathrm{~d} x_{2} \\
& =\int_{t}^{\infty} \int_{t(1-\rho) / \sqrt{1-\rho^{2}}}^{\infty}\left(\sqrt{1-\rho^{2}} y+\rho x_{2}\right) \varphi(y) \varphi\left(x_{2}\right) \mathrm{d} y \mathrm{~d} x_{2} \\
& =\int_{t}^{\infty} \sqrt{1-\rho^{2}} \varphi\left(\frac{t-\rho x_{2}}{\sqrt{1-\rho^{2}}}\right) \varphi\left(x_{2}\right)+\rho x_{2} \Psi\left(\frac{t-\rho x_{2}}{\sqrt{1-\rho^{2}}}\right) \varphi\left(x_{2}\right) \mathrm{d} x_{2} \\
& =\left(1-\rho^{2}\right) \varphi(t) \Psi\left(t \sqrt{\frac{1-\rho}{1+\rho}}\right)+\int_{t}^{\infty} \rho x_{2} \Psi\left(\frac{t-\rho x_{2}}{\sqrt{1-\rho^{2}}}\right) \varphi\left(x_{2}\right) \mathrm{d} x_{2} .
\end{aligned}
$$

Integration by parts, (10), and (12) finally yield

$$
\begin{aligned}
\int_{t}^{\infty} & \rho x_{2} \Psi\left(\frac{t-\rho x_{2}}{\sqrt{1-\rho^{2}}}\right) \varphi\left(x_{2}\right) \mathrm{d} x_{2} \\
& =\rho \varphi(t) \Psi\left(t \sqrt{\frac{1-\rho}{1+\rho}}\right)+\int_{t}^{\infty} \frac{\rho^{2}}{\sqrt{1-\rho^{2}}} \varphi\left(\frac{t-\rho x_{2}}{\sqrt{1-\rho^{2}}}\right) \varphi\left(x_{2}\right) \mathrm{d} x_{2} \\
& =\left(\rho+\rho^{2}\right) \varphi(t) \Psi\left(t \sqrt{\frac{1-\rho}{1+\rho}}\right) .
\end{aligned}
$$

Finally, using identities (10)-(12) and

$$
P_{t}(\rho)=\int_{t}^{\infty} \int_{t}^{\infty} \frac{1}{\sqrt{1-\rho^{2}}} \varphi\left(\frac{x_{1}-\rho x_{2}}{\sqrt{1-\rho^{2}}}\right) \varphi\left(x_{2}\right) \mathrm{d} x_{1} \mathrm{~d} x_{2}=\int_{t}^{\infty} \Psi\left(\frac{t-\rho x_{2}}{\sqrt{1-\rho^{2}}}\right) \varphi\left(x_{2}\right) \mathrm{d} x_{2},
$$

the results for $C_{t}(\rho)$ and $V_{t}(\rho)$ can be verified similarly; see [3].

\subsection{Proof of Theorem 2}

As $\varphi(0,0, \rho)=(2 \pi)^{-1}\left(1-\rho^{2}\right)^{-1 / 2}$ and

$$
\mathrm{P}(Z(o) \geq 0, Z(r) \geq 0)=\frac{1}{2 \pi}\left(\arcsin (R(r))+\frac{\pi}{2}\right),
$$

the formulae for $E$, cov, $\gamma$, and cor follow immediately from Lemma 1. Finally, $\bar{m}=$ $\mathrm{E}[Z(o) \mid Z(o) \geq 0]=\sqrt{2 / \pi}$ yields the result for $k_{m m}$. 


\section{Acknowledgement}

Zakhar Kabluchko was supported by DFG-SNF FOR 916 'Statistical Regularisation and Qualitative Constraints'.

\section{References}

[1] AdLer, R. J. (1981). The Geometry of Random Fields. John Wiley, Chichester.

[2] Adler, R. J. AND TAYlor, J. E. (2007). Random Fields and Geometry. Springer, New York.

[3] Ballani, F., Kabluchko, Z. and Schlather, M. (2012). Random marked sets. Preprint. Available at http://arxiv.org/abs/0903.2388v2.

[4] Beneš, V. and RataJ, J. (2004). Stochastic Geometry: Selected Topics. Kluwer, Boston, MA.

[5] Cramér, H. and Leadbetter, M. R. (1967). Stationary and Related Stochastic Processes. Sample Function Properties and Their Applications. John Wiley, New York.

[6] Cressie, N. A. C. (1993). Statistics for Spatial Data. John Wiley, New York.

[7] Cressie, N., Frey, J., Harch, B. and Smith, M. (2006). Spatial prediction on a river network. J. Agricultural Biol. Environ. Statist., 11, 127-150.

[8] Daley, D. J. And Vere-Jones, D. (2008). An Introduction to the Theory of Point Processes, Vol. II. Springer, New York.

[9] Diggle, P. J., Menezes, R. and Su, T.-L. (2010). Geostatistical inference under preferential sampling. J. R. Statist. Soc. C 59, 191-232.

[10] Diggle, P. J., Ribeiro, P. J., JR. And Christensen, O. F. (2003). An introduction to model-based geostatistics. In Spatial Statistics and Computational Methods (Aalborg, 2001; Lecture Notes Statist. 173), ed. J. Møller, Springer, New York, pp. 43-86.

[11] Guan, Y., Sherman, M. and Calvin, J. A. (2004). A nonparametric test for spatial isotropy using subsampling. J. Amer. Statist. Assoc. 99, 810-821.

[12] Hug, D., LAst, G. AND WeIL, W. (2004). A local Steiner-type formula for general closed sets and applications. Math. Z. 246, 237-272.

[13] Illian, J., Penttinen, A., Stoyan, H. and Stoyan, D. (2008). Statistical Analysis and Modelling of Spatial Point Patterns. John Wiley, Chichester.

[14] Kangas, A. and Maltamo, M. (2006). Forest Inventory: Methodology and Applications. Springer, Dordrecht.

[15] Kraynik, A. M. (1988). Foam flows. Ann. Rev. Fluid Mech. 20, 325-357.

[16] Liski, J. and Westman, C. J. (1997). Carbon storage in forest soil of Finland. Biogeochemistry 36, $239-260$.

[17] Matheron, G. (1969). Théorie des ensembles aléatoires. In Les Cahiers du Centre Morphologie Mathematique de Fontainebleau, Fasc. 4, Ecole des Mines de Paris.

[18] Matheron, G. (1975). Random Sets and Integral Geometry. John Wiley, New York.

[19] Molchanov, I. (1983). Labelled random sets. Teor. Veroyat. Matem. Statist. 29, 93-98 (in Russian). English translation: Theory Prob. Math. Statist. 29 (1984), 113-119.

[20] Molchanov, I. (2005). Theory of Random Sets. Springer, London.

[21] Pólya, G. (1949). Remarks on characteristic functions. In Proc. Berkeley Symp. on Mathematical Statistics and Probability, ed. J. Neyman, University of California Press, pp. 115-123.

[22] Sasvári, Z. (1994). Positive Definite and Definitizable Functions. Akademie, Berlin.

[23] Schlather, M. (2001). On the second-order characteristics of marked point processes. Bernoulli 7, 99-117.

[24] Schlather, M., Ribeiro, P. J., JR. And Diggle, P. J. (2004). Detecting dependence between marks and locations of marked point processes. J. R. Statist. Soc. B 66, 79-93.

[25] Serra, J. (1982). Image Analysis and Mathematical Morphology. Academic Press, London.

[26] Stoyan, D., Kendall, W. S. and Mecke, J. (1995). Stochastic Geometry and Its Applications. John Wiley, Chichester.

[27] TAKaнAтA, H. (1994). Nonparametric density estimation for a class of marked point processes. Yokohama Math. J. 41, 127-152.

[28] Wälder, O. ANd Stoyan, D. (1996). On variograms in point process statistics. Biometrical J. 38, 895-905.

[29] Wallerman, J., Joyce, S., Vencatasawmy, C. P. and Olsson, H. (2002). Prediction of forest stem volume using kriging adapted to detected edges. Canad. J. Forest Res. 32, 509-518. 